\title{
Transatlantica
}

Revue d'études américaines. American Studies Journal

1 | 2013

Revisiting the Gilded Age / Littérature et philosophie

\section{La formule ou comment la philosophie l'attrape}

\section{Richard Pedot}

\section{(2) OpenEdition}

Journals

Édition électronique

URL : https://journals.openedition.org/transatlantica/6361

DOI : 10.4000/transatlantica.6361

ISSN : 1765-2766

Éditeur

Association française d'Etudes Américaines (AFEA)

Référence électronique

Richard Pedot, «La formule ou comment la philosophie l'attrape », Transatlantica [En ligne], 1 | 2013, mis en ligne le 13 février 2014, consulté le 01 février 2023. URL : http://journals.openedition.org/ transatlantica/6361; DOI : https://doi.org/10.4000/transatlantica.6361

Ce document a été généré automatiquement le 1 février 2023.

\section{(c) (i) (9)}

Creative Commons - Attribution - Pas d'Utilisation Commerciale - Pas de Modification 4.0 International - CC BY-NC-ND 4.0

https://creativecommons.org/licenses/by-nc-nd/4.0/ 


\title{
La formule ou comment la philosophie l'attrape
}

\author{
Richard Pedot
}

Dans le paragraphe introductif de son « Bartleby, ou la formule », Gilles Deleuze écrit :

Il [Bartleby] ne veut dire que ce qu'il dit, littéralement. Et ce qu'il dit et répète, c'est JE PRÉFÉRERAIS NE PAS, I would prefer not to. C'est la formule de sa gloire, et chaque lecteur amoureux la répète à son tour. Un homme maigre et livide a prononcé la formule qui affole tout le monde. (Deleuze, 1993, 89)

2 Ainsi la lecture semble-t-elle commencer par la suggestion que l'amour de la sagesse n'est pas très éloigné de l'amour porté à une formule que l'on nommera, provisoirement, littéraire. Cela, ce rapprochement qui n'annule pas l'éloignement, sera l'objet du travail qui suit, avec le souci de comprendre comment la philosophie, à l'instar des collègues et de l'employeur du scribe, attrape et répète la formule. J'aimerais aborder la question par le biais de la lecture qu'un autre philosophe, Jacques Rancière, fait non de la nouvelle melvillienne mais du texte deleuzien, sous la forme d'une nouvelle répétition non sans intérêt, comme en témoigne son titre: «Deleuze, Bartleby et la formule littéraire » (Rancière, 1998). De «Bartleby, ou la formule » à ce titre, il y a plus d'un changement significatif. La substitution de « et » à « ou » dans une conjonction ternaire indique qu'il s'agit d'examiner une relation entre trois termes sans alternative. Mais c'est à se demander si, dans cet agencement, le deuxième terme n'est pas qu'un simple prétexte pour traiter de la question «Deleuze et la formule littéraire ». Quoi qu'il en soit, remarquons également que Rancière précise la formule par un adjectif qui ne qualifie au mieux qu'indirectement la formule chez Deleuze, pour qui elle se situe dans une matérialité (une «littéralité ») qui fait problème pour Rancière.

Mais ces deux lectures, bien qu'elles ne soient pas de même degré (Rancière ne commente pas la nouvelle), ont un intérêt autre que celui de pouvoir éventuellement distinguer deux philosophies. Cette dernière question n'est pas centrale ici, même s'il est inévitable d'y faire allusion. En me penchant sur la contamination de la philosophie, au premier degré (Deleuze) ou au deuxième degré (Rancière), j'entends plutôt réfléchir sur la capacité qu'a le texte melvillien de surgir, tel le personnage éponyme sur le seuil 
de l'étude notariale, dans les parages de la philosophie. Entrent en jeu ici, bien sûr, les liens tissés entre l'œuvre de Melville et la pensée philosophique ainsi que la porosité des frontières entre deux types d'écriture (dans le cas de figures contemporaines comme Thoreau ou Emerson). Mais au-delà des correspondances observables et souvent répertoriées par la critique melvillienne, il m'importe plutôt de souligner que "Bartleby " met en scène la lecture philosophique autour de l'énigme d'une formule contagieuse et constitue de ce fait une aventure de pensée particulière : une critique de la critique, au miroir de la fiction.

\section{Portrait du philosophe en récitant amoureux}

Gilles Deleuze n'est pas, on le sait, le premier à s'attarder à cette formule. Il ne manque pas d'ailleurs de mentionner Blanchot qui avait avant lui attiré l'attention sur elle comme expression de «la préférence négative, la négation qui annule la préférence et s'efface en elle » (Blanchot, 1980, 219). Mais si Blanchot l'a précédé en isolant l'énoncé melvillien en quelque sorte comme un composant tenant à la fois de la littérature et de la philosophie, Deleuze semble lui accorder plus d'autonomie encore : si on l'en croit, c'est bien «I would prefer not to » qui « affole tout le monde », et non le personnage. L'insistance sur la littéralité s'entend par opposition à toute lecture métaphorique, comme annoncé dès la première ligne: "Bartleby n'est pas une métaphore de l'écrivain, ni le symbole de quoi que ce soit.» (Deleuze, 1993, 89) Mais c'est aussi la marque d'un intérêt pour sa performativité, comme le note Rancière en d'autres termes: Deleuze « décrit volontiers l'œuvre come le développement d'une formule: une opération matérielle qu'accomplit la matérialité d'un texte » (Rancière, 1998, 179). D'où sans doute la disparition quasi instantanée de son énonciateur.

5 Non pas que la formule se substitue radicalement au personnage, bien qu'elle " agisse " alors que lui préférerait toujours ne pas avoir à le faire. Pour que ce fût le cas, il faudrait évidemment qu'elle possède des attributs d'un tel statut, accordés à Bartleby, même a minima. Il paraît plus juste de dire qu'elle fonctionne comme un adjuvant pour le «héros », et que c'est donc sa "magie " qui séduit le philosophe. Cependant, «I would prefer not to " n'est pas un simple « Sésame, ouvre-toi!", qui s'épuise dans sa fonction et se rangerait tranquillement dans une catégorie narratologique étanche. Deleuze se passionne davantage pour ses effets et la manière dont ils sont produits, ce qui signifie également regarder de près sa structure, pour affirmer que la formule « ravage le langage » et le confronte au silence, l'y faisant basculer tout entier (Deleuze, 1993, 94). Une telle proposition, fidèle à la théorie des devenirs qui innerve la philosophie deleuzienne, ne s'accorde pas, il est facile de le voir, avec une conception de la stabilité des structures langagières.

6 En fait, Deleuze va s'ingénier, quitte à forcer le trait, à montrer l'incomplétude foncière de la formule d'un point de vue linguistique, allant jusqu'à évoquer une forme d'agrammaticalité. C'est une des thèses fortes de la lecture proposée, qui ne sera d'ailleurs pas remise en cause par les commentateurs ultérieurs que sont Giorgio Agamben ou Jacques Derrida. Même Rancière, malgré les critiques formulées, ne reviendra pas sur cette affirmation, alors même qu'elle aurait pu servir ses arguments. Il n'en est que plus important de s'y attarder, d'autant que la démarche de Deleuze en la matière est frappée d'ambiguïté, d'une sorte qui laisse pressentir la force d'un désir - un désir de convaincre. 
Que dit-il précisément? Quel est son cheminement ? Cherchant à cerner la littéralité de la formule, Deleuze, dans un premier temps, pointe à la fois sa grammaticalité et son caractère bizarre :

la bizarrerie de la formule déborde le mot lui-même: certes, elle est grammaticalement correcte, syntaxiquement correcte, mais sa terminaison abrupte, NOT TO, qui laisse indéterminé ce qu'elle repousse, lui confère un caractère radical, une sorte de fonction-limite. Sa reprise et son insistance la rendent d'autant plus insolite, toute entière. Murmurée d'une voix douce, patiente, atone, elle atteint à l'irrémissible, en formant un bloc inarticulé, un souffle unique. À ces égards, elle a la même force qu'une formule agrammaticale. (Deleuze, 1993, 89)

8 Si la formule est correcte, pourrait-on dire, les choses devraient s'arrêter là, en toute rigueur linguistique. D'ailleurs, Deleuze remarque : «Les linguistes ont analysé ce qu'on appelle "agrammaticalité" en toute rigueur" (Deleuze, 1993, 89). Il mentionne plusieurs exemples de telles "anomalies", dont le fameux vers de Cummings, "he danced his did». Rien de cela ne correspond à première vue à l'énoncé de Bartleby, mais l'argument n'est pas aussi paradoxal qu'il y paraît. D'une part, aux yeux de Deleuze, le plus petit dénominateur commun est le concept de limite d'une série. La formule agrammaticale, ainsi, "vaut comme la limite d'une série d'expressions correctes » et de même «I would prefer not to », est-il suggéré, pourrait être considéré comme « limite d'une série telle que "je préférerais ceci, je préférerais ne pas faire cela, ce n'est pas ce que je préférerais" ». On saisit mieux le raisonnement de Deleuze par cette paraphrase, car on pourrait faire remarquer que «je préférerais ne pas faire cela » traduit « en toute rigueur » "I would prefer not to ». Ce qui autorise à parler d'expression-limite est le fait que par la répétition (le «bégaiement» de la langue) la proposition tend à devenir intransitive, alors qu'elle n'est qu'elliptique à chaque occurrence en contexte. C'est donc bien à ses effets que l'on reconnaît la bizarrerie de la formule: "Malgré sa construction normale, elle résonne comme une anomalie.» (Deleuze, 1993, 90)

9 L'ambiguïté se conçoit mieux, dès lors. La formule de Bartleby est, tout autant que la formule agrammaticale, proposée comme limite d'une série d'«expressions grammaticales ordinaires ", mais l'équivalence n'est pas parfaite. Le second type de formule déroge d'emblée à la "correction » syntaxique et c'est cette incorrection qui renvoie à la norme, tandis que "I would prefer not to " est une expression correcte - «à la limite ». C'est-à-dire qu'on doit la considérer comme un point de bascule dans le langage qui met en évidence le devenir-agrammatical du langage ordinaire. Mais l'accent mis sur la limite, le devenir, dans chaque cas - accent qui est parfaitement cohérent dans la philosophie deleuzienne - est ce qui empêche de voir dans l'analogie un simple forçage. L'exemple de l'énoncé melvillien ajoute en fait quelque chose à l'hypothèse concernant les énoncés incorrects: point n'est besoin d'anomalie syntaxique pour affoler le langage, le soupçon y suffit et la possibilité de "résonner " par «stéréotypie » (Deleuze, 1993, 90), comme si une autre syntaxe était en cause. À rebours, on dira que la rigueur linguistique ou narratologique ne suffit pas à rendre compte des effets de langage, qu'elle peut même parfaitement passer à côté.

10 Mais que faut-il entendre par effets de la formule ? Sa singularité tient d'une part à ce qu'ils ne se limitent pas à sa performativité entendue au sens strict et d'autre part à ce qu'elle n'est pas réservée aux péripéties de l'intrigue.

11 Comme d'autres avant et après lui, Deleuze note le recours par l'homme de loi à la doctrine des assumptions ("références ou présupposés», dans sa traduction), et 
remarque : «L'avoué fait lui-même la théorie des raisons pour lesquelles la formule de Bartleby ravage le langage. »(Deleuze, 1993, 94) Mais il déplie immédiatement sa propre théorie de la référence en en distinguant deux types, à savoir les propositions constatives et les speech acts qui sont auto-référentiels et «assurent un rapport avec l'interlocuteur " (Deleuze, 1993, 95). Il ne s'agit pas d'une opposition qui privilégierait le performatif au sens strict contre le constatif. En effet, pour Deleuze, parler suppose " ce double système de références", car parler c'est toujours accomplir des actes " qui assurent un rapport avec l'interlocuteur, suivant nos situations respectives » (Deleuze, 1993, 95). La formule de Bartleby "ravage » ce double système. Dans le système constatif, en l'absence d'alternative entre un mot et les autres, " elle creuse une zone d'indétermination qui fait que les mots ne se distinguent plus» (Deleuze, 1993, 95). Ce serait là, supposera-t-on, son caractère intransitif. Mais dans le même temps, performativement, et de manière qui paraît plus dévastatrice, « elle désamorce les actes de paroles d'après lesquels un patron peut commander, un ami bienveillant poser des questions, un homme de foi promettre ». C'est dès lors par ce double effet, en minant tous les présupposés du langage, que « malgré son air correct, [elle] fonctionne comme une véritable agrammaticalité » (Deleuze, 1993, 95).

Là réside l'attrait principal de la formule aux yeux de Deleuze et on peut penser que sa semi-agrammaticalité n'y est pas pour rien dans la mesure où par sa "correction " apparente elle investit toujours déjà le langage de l'intérieur. L'allusion à la lecture faite par l'homme de loi néanmoins appelle quelques commentaires. Deleuze en fait greffe la sienne sur celle-ci afin d'en corriger l'erreur de parallaxe, pour ainsi dire. Répondant toujours à l'énigme-Bartleby " par la charité, la philanthropie, tous les masques de la fonction paternelle» (Deleuze, 1993, 112-113), affolé qu'il est par "l'entêtement d'une pensée sans image, d'une question sans réponse, d'une logique extrême et sans rationalité » (Deleuze, 1993, 106), et « le devenir où Bartleby, par sa seule existence, risque de l'entraîner » (Deleuze, 1993, 113), l'homme de loi est délogé de toutes ses prémisses (ou plus exactement premises), qu'elles soient d'ordre professionnel (légal), philosophique ou moral. Sans entrer dans le détail des différences entre les deux lectures, on constatera de l'une à l'autre une inversion de polarité : là où l'homme de loi, «an eminently safe man », ne verrait que risque de désordre, Deleuze identifie une chance: une déterritorialisation de la loi paternelle, une issue au combat des fils schizos contre les pères, y compris les pères bienveillants qui au bout du compte finiraient par choisir la loi et la raison. On en conclura que les effets de la formule sont avant tout sensibles à partir du constat des forces en présence. C'est pourquoi il importe peu que la formule soit à proprement parler agrammaticale, il suffit qu'elle puisse faire apparaître et faire fuir le paradigme grammatical sur lequel elle s'enlève : "I PREFER NOT TO est aussi un trait d'expression qui contamine tout, s'échappant de la forme linguistique, destituant le père de sa parole exemplaire, autant que le fils de sa possibilité de reproduire ou de copier. » (Deleuze, 1993, 100)

Cette défiance de la grammaire a des côtés nietzschéens, et elle signifie qu'il ne faut pas entendre le concept dans les limites de la linguistique. L'agrammaticalité en cause dans la formule contamine l'ensemble du texte comme instrument de déterritorialisation du générique. En témoigne l'allusion au roman anglais et à Dickens :

Tout commence comme dans un roman anglais, à Londres et chez Dickens. Mais quelque chose d'étrange se produit chaque fois, qui brouille l'image, la frappe d'une incertitude essentielle, empêche la forme de "prendre ", mais aussi défait le sujet, le jette à la dérive et abolit toute fonction paternelle. [...] Tout commençait à 
l'anglaise, mais on continue à l'américaine, suivant une ligne de fuite irrésistible.

(Deleuze, 1993, 99) littérature américaine, ainsi qu'on peut le voir quelques pages plus haut : « N'est-ce pas notamment la vocation schizophrénique de la littérature américaine, de faire filer ainsi la langue anglaise, à force de dérives, de déviations, de détaxes ou de surtaxes (par opposition à la syntaxe standard) ? Introduire un peu de psychose dans la névrose anglaise ? Inventer une nouvelle universalité ?» (Deleuze, 1993, 93) ${ }^{1}$ La formule de la formule serait donc la déterritorialisation de la littérature anglaise par la littérature américaine. En dépit du schématisme de cette géopolitique littéraire, et du traitement expéditif de la figure paternelle de Dickens ${ }^{2}$, l'intuition est juste. En effet, il est possible de voir « Bartleby » comme l'histoire d'une déroute (un homme de loi désemparé face à son employé) qui est également la déroute d'une histoire, celle qu'il aimerait bien mais ne peut raconter. En ce sens effectivement, la formule est ce qui empêche la forme (celle du grand roman anglais, représenté par Dickens) de prendre et la fait filer. De plus, on notera dans sa performativité, même si Deleuze ne s'y arrête pas, un paramètre non négligeable qui a trait à l'héritage puritain et se traduit par un questionnement sur le pouvoir des signes. À ce titre, d'ailleurs, le «I would prefer not to » de Bartleby n'est pas si éloigné du voile de crêpe noir porté par le révérend Hooper dans « The Minister's Black Veil» de Hawthorne, lequel affole également la communauté qui entoure le personnage principal, fait proliférer les interprétations et laisse tous face à un mystère insondable ${ }^{3}$. Encore une fois cependant, il faut signaler que l'interprétation deleuzienne ne s'inscrit pas dans une approche énonciative classique : les mots de Bartleby ne sont pas équivalents à un fiat de quel qu'ordre que ce fût, leur effet est en quelque sorte inverse, ils bouleversent le pouvoir du langage (légal, moral, ...- paternel, pour dire vite) en y introduisant de l'incalculable.

Voici donc, en résumé, ce qui fait que Deleuze attrape la formule. En premier lieu, sa philosophie est séduite par sa quasi-agrammaticalité, comme accès au devenir et à la déterritorialisation du langage par le langage. Cette séduction est la plus explicitement reconnue. La littérature fait signe à une philosophie à l'affût des processus de minoration de la langue majeure qui définissent la littérature $e^{4}$. La philosophie y reconnaît ses enjeux, s'y reconnaît, et y teste ses concepts et il n'est plus aisé de décider si la formule est d'abord littéraire ou philosophique, habitant le seuil entre les deux domaines. Objecter que la philosophie ne fait que traduire les figures en concepts est une simplification, car elle ne peut dire quelque chose à leur propos qu'en acceptant de s'y perdre. À l'appui de cette hypothèse, il serait possible de montrer comment la langue de Deleuze est « contaminée » par l'expression littéraire, se manifestant souvent par une forme d'« insistance adjectivale » (pour reprendre l'expression de F. R. Leavis à l'encontre de Joseph Conrad), là où semble-t-il le concept ne peut suffire. Mais il suffit de remarquer que la poéticité de la formule - Deleuze évoque l'« expression hautement poétique de Melville» (Deleuze, 1993, 90) - vient appuyer l'argument en faveur de l'anomalie de la construction. En somme, la formule entre en philosophie grâce à son caractère éminemment poétique, et non en dépit de celui-ci.

Nous retrouvons ici la citation de départ. Voir d'entrée la formule sous l'angle de la séduction amoureuse, c'est se placer du côté du percept et de l'affect ${ }^{5}$ et déborder les schémas classiques de la critique. Soulignant l'effet de la formule sur son lecteur, le philosophe met en avant la porosité de la frontière avec l'objet-texte : la contagion de la formule n'est pas qu'un des éléments de l'intrigue, elle existe également dans le 
rapport du lecteur au texte. La porosité en question favorise la contagion en même temps qu'elle la met en abyme, c'est l'exemplarité de la formule de Bartleby. La philosophie deleuzienne ne peut pas ne pas «l'attraper »-comme on attrape un virus ou comme on se saisit d'un concept.

Mais la fascination ne tient pas seulement à la structure et à la position indécises de la formule. Elle s'exerce à un niveau moins explicite et non théorisé par Deleuze. La formule s'attrape par un autre de ses tours et retours, où la philosophie se trouve dans une position qui ressemble à une forme de déni. C'est le deuxième point qui se déduit de tout ce qui précède. Si Deleuze répète bel et bien l'énoncé de Bartleby, preuve qu'il a été contaminé comme l'entourage du scribe, sa répétition, pas plus que celle de l'homme de loi ou de ses employés, n'équivaut à celle de Bartleby. La distinction est essentielle. La syntaxe des réitérations de Bartleby est comme lui-même insondable (" inscrutable »), même s'il reste encore beaucoup à dire de ses variations - dont les commentaires philosophiques ont peu cure. Elle diffère en tout cas des reprises par les autres clercs dont les propos sont toujours d'une manière ou d'une autre solidaires des prémisses de leur monde professionnel. Même le singulier et menaçant «I'd prefer him » de Nippers, qui frise l'anomalie, exprime bien en contexte le souhait que Bartleby soit réinséré dans la longue chaîne de production de signifiants qu'est l'office notarial - ne serait-ce que pour ne plus avoir à copier ou collationner à sa place.

18 L'homme de loi n'échappe pas non plus à la répétition, à distinguer également de la répétition de Bartleby dans la mesure où elle semble animée par un désir de comprendre: c'est le seul à s'interroger sur son sens et sa syntaxe (son histoire) possibles et à les confronter ouvertement à des systèmes d'explication, autres que le système légal dont il sent bien l'inadéquation en l'occurrence. Deleuze le reconnaît implicitement en indiquant qu'il fait la théorie du ravage de la formule en rangeant le personnage parmi les « Témoins, [...] récitants, [...] interprétants » (Deleuze, 1993, 104), mais c'est toujours en pointant l'insuffisance de sa position, du point de vue philosophique. N'est-ce pas congédier un personnage essentiel un peu trop rapidement, comme s'il n'était qu'un moyen commode de suggérer la supériorité de sa propre lecture? Non pas que les arguments concernant les limitations des explications proposées par le "témoin » ne soient pas convaincants, mais ils permettent de ne pas s'interroger sur sa propre position, car le philosophe, comme tout lecteur du récit, est témoin et interprétant, et également récitant dès qu'il répète la formule. Tout se passe comme si, pour Deleuze, la répétition philosophique ne pouvait être qu'amoureuse, c'est-à-dire du point de vue de l'énonciateur premier. C'est oublier, au moins, que cette énonciation, malgré les apparences, est déjà affectée de supplémentarité par le récit et commentaire du témoin-narrateur.

D'où le coup de force de l'affirmation: Bartleby «ne veut dire que ce qu'il dit, littéralement. Et ce qu'il dit et qu'il répète, c'est JE PRÉFÉRERAIS NE PAS, I would prefer not to. " (Deleuze, 1993, 89) L'homme de loi, lui, ne l'entend pas ainsi. Il fait littéralement l'expérience de l'efficace de la formule, mais cherche à y trouver un sens, une justification. Dans la parenthèse de la fiction qui laisse la référence suspendue et le mystère tout entier, on ne peut lui donner tort ni raison, mais son lecteur, Deleuze, tombe dans un piège semblable à celui où il semble le voir enfermé. Si Bartleby veut dire quelque chose, quoi que ce fût, il n'est plus dans la préférence négative dont tout système de référence, de vouloir-dire, est banni. À moins que ce ne soit ici le vouloirdire de Deleuze. Prendre le parti apparent de répéter la formule avec le schizo, le 
messie, «le médecin d'une Amérique malade » (Deleuze, 1993, 114), plutôt qu'avec le névrosé, homme de loi ou psychanalyste ${ }^{6}$, revient à refouler la part névrotique de toute interprétation, même la plus déconstruite. En fin de compte, la répétition philosophique ne serait-elle pas plutôt celle de l'homme de loi, du père bienveillant mais destructeur? La question n'est en fait pas tranchée et cela ne rend la formule que plus séduisante, car elle joue à la fois de ces deux possibilités et ce d'autant plus que l'homme de loi lui-même est gagné sans doute par une répétition amoureuse ${ }^{7}$. Quant à ce dernier point, en effet, de manière significative, la position du narrateur n'est pas moins ambivalente que celle du philosophe.

En effet, malgré la défiance professée à l'égard de l'imagination poétique (avec «the mettlesome poet Byron » (67) comme repoussoir de choix), la narration se voit souvent contaminée par son dehors : le devenir-poétique du langage de la loi. Considérons, par exemple, le moment où l'employeur de Bartleby envisage de le chasser: "Then something severe, something unusual must be done. What! surely you will not have him collared by a constable, and commit his innocent pallor to the common jail? » (91) L'inhabituel, en l'occurrence, est déjà que le poétique (l'hypallage " his innocent pallor ») s'est insinué dans une expression de type juridique. Mais c'est qu'en fait la loi et le langage qui la supporte (syntaxe et lexique) sont délogés de leurs premises. Impossible en effet d'enfermer pour vagabondage celui qui reste sur place ou qui indiscutablement a des moyens de subsistance («indubitably he does support himself »). L'immobilisme du scribe est comparable à sa formule. Il n'a rien d'incorrect, vis-à-vis de la loi, mais sa performativité la dé-range au point qu'elle doit changer de premises pour pouvoir en faire un délinquant ordinaire : «Since he will not quit me, I must quit him. I will change my offices; I will move elsewhere; and give him fair notice, that if I find him on my new premises I will then proceed against him as a common trespasser. » On constate également que la préférence négative a contaminé l'homme de loi, qui préférerait ne pas chasser Bartleby.

Voici donc un autre biais par lequel le philosophe attrape la formule, selon son envers. Non plus par l'approbation de son pouvoir déterritorialisant, de sa force messianique, mais par le déni de l'ambivalence de la répétition-interprétation du narrateur, déni qui englobe aussi l'ambivalence de sa propre lecture. Dit autrement, une trop grande proximité avec l'homme de loi, malgré les différences évidentes, serait aussi puissante en retour à faire dériver la philosophie vers les lignes de fuite schizophrénique qu'une célébration amoureuse de ces dernières. On ne peut penser la formule sans l'arrièreplan duquel elle « se détache », donc sans en penser la « récitation » par l'homme de loi, ni la reproduire d'une manière ou d'une autre.

On se gardera néanmoins de lire uniquement à travers le filtre de ces deux personnages, même en soulignant l'oscillation possible entre les perspectives qu'ils nomment. Car c'est une des forces et un des grands attraits de la formule que de remettre en cause la position de tout énonciateur. Nous avons déjà remarqué qu'elle paraît plus forte que celui qui l'a énoncée en premier. Deleuze résume : « un homme maigre et livide a prononcé la formule qui affole tout le monde ». Mais, dira-t-on, pourquoi préciser que l'énonciateur est un homme maigre et livide? En quoi cela pourrait-il concerner la puissance de la formule? Le philosophe ne s'en explique pas. Un rapprochement est possible avec l'idée de l'« irrésistible petite santé de l'écrivain » qui l'ouvre à "des devenirs qu'une grosse santé dominante rendrait impossibles " (Deleuze, 1993, 14), mais c'est le statut évanescent, spectral, de Bartleby qui importe le 
plus ici. La formule est d'autant plus puissante qu'elle emporte son énonciateur dans le devenir-autre du langage, où il peut devenir in-différent. Deleuze en effet insistera sur l'homme "sans qualités, sans particularités " qu'est le scribe, trait qui en fait potentiellement un messie: un homme "dont on attend, peut-être, qu'il en sorte l'Homme de l'avenir ou d'un nouveau monde » (Deleuze, 1993, 96).

La position spectrale de Bartleby, « la puissance de l'impersonnel » (Deleuze, 1993, 13), serait encore un autre facteur de contamination. Tout lecteur, témoin, récitant, interprétant, peut tenter et être tenté de se substituer au scribe sans être pour autant, pas plus que lui, le véritable énonciateur d'une formule proprement affolante. Il faudrait par ailleurs prendre le temps d'interroger en parallèle l'influence que peut avoir en la matière le caractère hautement poétique de la formule, et se demander si la poéticité - opposée à la narrativité - n'est pas un autre nom pour la littéralité et la performativité. C'est la question des rapports entre poésie et philosophie dans la philosophie contemporaine qui est en jeu ici, question qui n'est sans doute pas dissociable de celle des rapports entre poésie et roman ou mieux peut-être entre poétique et romanesque. Essayer seulement de la poser nous entraînerait bien au-delà du cadre de cet article et je ne m'y risquerai pas, mais nous la retrouverons obliquement entre Deleuze et Rancière.

\section{Rancière change la / de formule}

Le texte de Rancière peut être abordé de différentes façons. L'une consisterait à le prendre comme une critique de la lecture deleuzienne de "Bartleby », c'est-à-dire en dernière analyse comme une autre lecture philosophique du conte. L'autre y verrait une critique de la philosophie et de la poétique de Deleuze, où «Bartleby » n'est qu'un prétexte commode. À l'appui de cette deuxième possibilité, la plus vraisemblable, on se rappellera son intitulé et le propos introductif qui lit le titre de Deleuze comme un condensé du " mode de lecture des œuvres » propre au philosophe, qui « voit volontiers l'œuvre comme le développement d'une formule: une opération matérielle qu'accomplit la matérialité d'un texte" (Rancière, 1998, 179). Comme Rancière ne prétend à aucun moment s'intéresser particulièrement à "Bartleby ", il devrait s'en déduire qu'il n'y a pas lieu de chercher une lecture philosophique du texte melvillien dans son texte, ni même de prendre le temps de nous y intéresser en dehors de sa pertinence philosophique. Si nous nous y arrêtons malgré tout, ce n'est pas prioritairement pour l'éclairage incident qu'il pourrait malgré tout apporter au texte de fiction. C'est surtout qu'il nous paraît exemplaire de l'extension philosophique de la formule même dans un texte qui se place à distance.

Se dessine donc une troisième manière de lire Rancière qui ne viserait ni à rectifier ce qu'il y aurait à rectifier dans l'interprétation de la fiction, ni à intervenir dans le différend entre deux philosophies mais plutôt à estimer ce qui est en jeu, quant aux rapports entre philosophie et littérature, dans le choix d'une formule - et de cette formule littéraire en particulier - pour en découdre ${ }^{8}$. Le choix de Rancière, en effet, est loin d'être simple. Pourquoi, en premier lieu, s'arrêter exclusivement sur la formule ou, mieux, sur la formule "condensée " qu'est le titre de Deleuze - «Ce titre condense bien le mode de lecture qui lui est propre.» (Rancière, 1998, 179) De la sorte, le commentateur répète la réduction de la nouvelle à un de ses traits saillants, de manière 
critique certes mais en l'aggravant considérablement puisqu'il ne retourne pratiquement pas à la fiction considérée.

Pourquoi, ensuite, choisir cette formule précise plutôt que celles mises en avant dans un autre texte de Critique et clinique, intitulé «Sur quatre formules poétiques qui pourraient résumer la philosophie kantienne »? L'usage qu'en fait Deleuze semble pourtant plus directement corroborer la critique du recours à la formule comme emblème malgré l'insistance (dans «Bartleby » en particulier) sur la littéralité et la performativité de l'œuvre littéraire. Une réponse à cela pourrait être que, précisément, il s'agit avant tout de pointer une aporie dans un texte où l'accent, à l'inverse de l'article cité, est placé sur la performativité contre le symbolisme. Le choix est donc parfaitement cohérent, mais alors nous devons nous poser la question de la performativité de l'énoncé de Bartleby dans son extension, et nous verrons que l'argument de Rancière à cet égard est limitatif et tombe en partie par manque de nuance.

En l'occurrence, et c'est le troisième point d'interrogation, Rancière lit Deleuze lisant la formule à travers le filtre d'une autre formule. Il s'agit de la non moins fameuse déclaration flaubertienne "il n'y a pas de beaux et de vilains sujets ». Gisèle Berkman résume : "Rancière tente de faire passer l'une dans l'autre la formule "indifférentiste" de Bartleby et la phrase de Flaubert proclamant l'égalité en droit des sujets » (Berkman, 2011, 117). Ce glissement, on le devine, ne va pas de soi et l'on peut déjà faire l'hypothèse qu'il permet de rendre compte des problèmes soulevés plus haut: la formule melvillienne, en tant que formule, s'y prête mieux que toute autre. En ce sens déjà, Rancière à son tour a attrapé la formule. Voici comment :

La formule de Bartleby accomplit ainsi en cinq mots un programme qui pourrait résumer la nouveauté même de la littérature. Et son énoncé même est étrangement proche de ceux qui définissent cette nouveauté. "Préférer ne pas» peut se paraphraser et s'interpréter de différentes manières, dont l'une est : « renoncer à préférer», "ne plus vouloir préférer». Dans cette version, elle devient formellement homologue à l'une des formules canoniques qui norment la volonté de littérature, je veux parler du principe flaubertien célèbre : il n'y a pas de beaux et de vilains sujets, pas de raisons de préférer Constantinople - c'est-à-dire les fastes de l'Orient et de l'Histoire - à Yvetot - c'est-à-dire l'humidité et la grisaille sans histoire de la province française. Il n'y en a pas car le style est une manière absolue de voir les choses. (Rancière, 1998, 180)

Ce paragraphe charnière, qui représente pratiquement le seul moment où Rancière propose une « lecture » de (la formule de) «Bartleby » suscite plusieurs commentaires sur la traduction de la formule. On notera d'abord que sont laissés de côté les propos de Deleuze sur la force de la formule et sa grammaticalité limite pour se concentrer sur sa sémantique, son côté "programmatique» - mais de quel programme s'agit-il au fond? - ou, pour emprunter au vocabulaire utilisé à propos de Deleuze, son côté emblématique. La performativité est mise à l'écart au profit de la réitération d'une "norme » littéraire ${ }^{9}$, comme disparaît également la modalité conditionnelle dans la substitution du syntagme verbal "préférer ne pas» à l'énoncé original. Mais les «traductions » qui découlent de ce glissement sont plus surprenantes et ne paraissent pouvoir se justifier que par le parallèle recherché avec le principe flaubertien. Le paradoxe de cette surtraduction est qu'elle serait mieux justifiée en conservant le conditionnel de l'original, qui modalise la préférence - tout en rendant cependant caduque toute idée de renoncement ou de volonté. L'ironie est de parler d'homologie formelle, là où la forme d'origine n'est même pas prise en compte, l'homologie 
n'existant que dans et pour la «version » rancièrienne. De ce point de vue, le coup de force interprétatif semble être plus du côté de Rancière que du côté de la littéralité deleuzienne, sauf à considérer que l'homologie se démontre ailleurs que dans la simple formule et que la nouvelle de Melville serait la formule de "l'indifférentisme " littéraire - ce que Rancière ne prend pas le temps de discuter.

Enfin, il est significatif qu'une équivalence soit créée entre une citation extraite d'une fiction et une autre qui est déjà un commentaire, tirée d'un échange épistolaire entre deux écrivains (Gustave Flaubert et Louise Colet). Un lien est donc installé entre une expression dramatique, issue d'un contexte et d'un agencement d'énonciation dont il n'est pas tenu compte, et un axiome à portée conceptuelle (ou «normative»). Il n'y a rien d'illégitime ou d'incohérent à ce type de parallèle. En la matière, Deleuze procède de façon comparable, puisqu'une grande partie de son argument repose sur une autre citation de statut comparable à celle de Flaubert, celle de Proust dans Contre SainteBeuve qui dit que les beaux livres sont écrits dans une sorte de langue étrangère. En revanche, le Contre Deleuze de Rancière est en porte-à-faux en ce sens qu'il veut trop prouver à partir de la formule flaubertienne, érigée en principe, et pas assez à partir du texte lu par Deleuze. Dès lors, même si certaines de ses critiques sont fondées, il convient de les relire en raison de ce biais.

Reprenons quelques grandes lignes du raisonnement. Pour Rancière, faire de la littérature le développement d'une formule équivaut chez Deleuze à une double opposition : d'un côté à l'intrigue aristotélicienne (position anti-narrative) et de l'autre au sens caché (position anti-symbolique). Cependant, selon lui, Deleuze ne tient pas ses positions et «la pureté de la "formule", dans le détail de l'analyse, est soumise à plus d'un va-et-vient entre les pôles refusés de l'histoire et du symbole " (Rancière, 1998, 179). Par exemple, on peut constater l'importance accordée à la figure du héros (Bartleby le messie, le sauveur de la société des frères), qui réintroduit donc le récit et privilégie le caractère au détriment de l'action. Ou encore Deleuze montre «le plus souvent » la puissance matérielle de la littérature « en nous disant non pas ce que la langue ou la forme opèrent mais ce que la fable nous raconte» (Rancière, 1998, 188-189).

31 La première remarque est juste dans son constat, mais l'hypothèse de Rancière est d'abord que l'abolition de « la hiérarchie des représentés » ruine " l'édifice mimétique » construit sur elle (Rancière, 1998, 189). De ce point de vue, Deleuze apparaît affairé à construire les mythes de ses héros ou les héros de ses mythes, bien loin de Flaubert, en effet. L'aporie est remarquable et Rancière a raison de s'y arrêter mais pourtant il ne semble pas que la formule flaubertienne suffise à épuiser le sujet, laissant croire que le héros deleuzien est comparable à celui d'un mythe au sens classique ou d'un récit aristotélicien et que Deleuze purement et simplement succombe à la mimésis d'action et reste prisonnier de la rhétorique. Dès lors, est minoré le rôle joué par le personnage conceptuel dans la théorie de la littérature deleuzienne, rôle lui-même aporétique en ce qu'il forme, dans la philosophie mais également dans la littérature, le point de passage entre figure et concept, n'étant à proprement parler ni l'un ni l'autre, ni une synthèse des deux. Comme j'ai tenté de le montrer ailleurs (Pedot, 2010), le personnage conceptuel deleuzien possède une hérédité chargée, à la fois dans la critique littéraire et dans la philosophie, qui complique la tâche de la philosophie. Cependant, on ne saurait oublier que, pour Deleuze, dans l'énonciation philosophique Je «[...] est toujours une troisième personne » (Deleuze et Guattari, 1991, 63) et un devenir, et 
qu'il met en garde contre la confusion entre philosophe et poète, thaumaturge, créateur de mythe, et entre personnages conceptuels et personnifications mythiques, personnes historiques ou héros littéraires (Deleuze et Guattari, 1991, 73). Cela ne met pas fin aux apories, qui sont constitutives de la notion même de personnage, mais l'aporie témoigne d'une tentative de réponse à la question « comment rendre visibles des forces invisibles ${ }^{10}$ ?", ce qui ne peut manquer de renvoyer à cet "incube» paradoxal : ce voyageur immobile qu'est Bartleby.

32 C'est cette question qu'écartent, en même temps que la performativité de "I would prefer not to ", les prémisses de Jacques Rancière qui font de la formule indifférentiste flaubertienne une formule princeps, excluant toute hiérarchie des sujets et des personnages. L'équivalence entre le commentaire flaubertien et la citation de l'œuvre de Melville revient à faire l'impasse sur le commentaire de Proust qui autorise en quelque sorte la lecture de Deleuze, ce même commentaire qui étaye opportunément pour lui ses hypothèses sur la langue littéraire, langue du dehors, quasi agrammaticale, impersonnelle et performative qu'il retrouve dans la formule de Bartleby. D'où de nouveau une critique en porte-à-faux par rapport aux tensions qu'elle repère justement.

Quand Rancière conteste la puissance matérielle de la formule en suggérant que «le plus souvent " Deleuze nous raconte ce que la fable dit plutôt qu'il nous montre comment la langue ou la forme opère, il faut reprendre son argument depuis son envers : si la formule de Bartleby «est bien littérale, et en même temps elle ne l'est pas " (Rancière, 1998, 188), alors la critique de Rancière est vraie et en même temps fausse. D'un côté, Deleuze ne pousse pas assez loin son hypothèse sur le bégaiement de la langue en regard de «Bartleby ». On ne saurait le faire sans examiner de près le texte de fiction pour sa littéralité, dans un autre sens du terme - son dispositif énonciatif particulier, sa figuralité, ses apories, le trajet de ses signifiants, sa poéticité. D'un autre côté, malgré l'ambivalence que nous avons notée, Deleuze n'a de cesse de rappeler la quasi-agrammaticalité de la formule qui explique, pour lui, son opérativité, qui n'est pas limitée à telle ou telle occurrence mais renvoie à une syntaxe proprement énigmatique à travers tout le texte. C'est pourquoi d'ailleurs il mentionne ses variantes - avec ou sans conditionnel, avec ou sans complément - pour conclure que même dans celles-ci « on sent la sourde présence de la forme insolite qui continue à hanter le langage de Bartleby " (Deleuze, 1993, 90), tandis que Rancière, paradoxalement pour son propos, ne s'arrête pas à la langue ou à la forme : il ne cite d'ailleurs qu'une seule fois au début « le célèbre I would prefer not to » (Rancière, 1998, 179) pour lui substituer rapidement les équivalents que l'on a vus, sans un commentaire sur sa construction.

Ici une difficulté de la démonstration de Deleuze est en cause mais c'est comme si Rancière attendait de la philosophie de ce dernier qu'elle lui donne ce qu'elle ne peut lui offrir: une solution à cette difficulté, à savoir un moyen de chiffrer grammaticalement le dérèglement de la langue. Dans la logique du devenir, «ce n'est plus la syntaxe formelle ou superficielle qui règle les équilibres de la langue, mais une syntaxe en devenir, une création de syntaxe qui fait naître la langue étrangère dans la langue, une grammaire du déséquilibre » (Deleuze, 1993, 141). Le bégaiement n'est donc pas nécessairement sensible à la surface formelle de l'énoncé, même si cela arrive (chez Artaud, par exemple), et la limite asyntaxique n'est pas extérieure au langage, elle en est le dehors. La demande que Deleuze adresse à la philosophie peut alors paraître exorbitante, elle l'est certainement aux yeux de Rancière. Elle consiste ni plus ni moins 
à donner acte à la littérature de sa forme insolite, quoique correcte, et de son sourd pouvoir de hanter le lecteur, y compris le philosophe.

L'objection de Rancière vise juste mais elle est injuste, car elle ne pointe pas les insuffisances de l'argumentation de Deleuze sur pièces, n'entrant pas dans le détail de ce que la langue de "Bartleby » fait, et à peine dans ce qu'elle dit puisqu'il n'évoque que la formule, dans une seule version, pour ce qu'elle dit selon lui. On observe d'ailleurs que Rancière corrige (c'est son mot) l'affirmation de Deleuze concernant la littéralité de la formule de Bartleby, mais utilise d'autres exemples (Gregor dans La Métamorphose, Isabelle dans Pierre, or the Ambiguities) ${ }^{11}$ à l'appui de sa démonstration. Mais ces exemples ne sont pas aussi probants que Rancière l'espérerait, comme on peut le constater à partir du premier.

Il est patent que, comme le souligne Rancière, le lecteur n'entend pas le piaulement douloureux de Gregor, illustration parfaite du dehors du langage pour Deleuze, mais la question qui se pose est de savoir si c'est ce dernier qui est naï de confondre le mot et la chose ou Rancière de croire ou feindre de croire que c'est de cela qu'il s'agit. Nous sommes conduits à nous demander ce que serait un piaulement que le lecteur pourrait entendre. En admettant même qu'une onomatopée ou un jeu typographique en soit un équivalent recevable, ce piaulement serait-il plus à même de « créer une autre langue dans la langue » (Rancière, 1998, 189) ? Là encore, il faut prendre le parti d'une hantise, de la hantise du piaulement, et dire, contre et avec Rancière, que le dit piaulement est symbolique (du pouvoir de la littérature) et en même temps ne l'est pas. Si Deleuze ne pousse pas plus loin l'examen du dérèglement de la langue dans La Métamorphose, il ne s'ensuit pas que ces hypothèses sont invérifiables - sauf à croire à la puissance du symbolisme et, chemin faisant, à ne lire Kafka que selon cette perspective ; sauf à croire également que La Métamorphose n'est que le récit d'une métamorphose, et d'une seule métamorphose, et reste indemne de toute contagion métamorphique.

La question se pose simplement: que fait le piaulement à la langue, si elle-même, littéralement, ne piaule pas? À suivre le raisonnement de Rancière : il ne fait rien, car « la langue de Kafka ne nous donne que la transcription de paroles que Grégoire émet et la notation de l'étrangeté par lui éprouvée de son timbre " (Rancière, 1998, 189). La partition serait donc trop bien réglée pour faire trembler la langue, mais c'est sans compter ses bougés les plus imperceptibles, à la limite. Rancière, une fois de plus lancé sur cette voie par Deleuze, ne s'arrête que sur une formule, le "piaulement douloureux", comme objet détaché. Mais même le contexte immédiat invite à introduire une nuance entre ce que «la langue» (de Kafka) dit et ce qu'elle fait. Si le piaulement irrépressible ( nicht zu unterdrückend») semble réprimé dans la langue qui l'évoque sans le faire entendre, comme indicateur de la métamorphose en cours, il est accompagné de phénomènes langagiers subreptices mais troublants.

Ainsi en va-t-il de l'emploi du pronom « es » juste avant l'allusion au piaulement :

Als er dies alles in größter Eile überlegte, ohne sich entschließen zu können, das Bett zu verlassen [...], klopfte es vorsichtig an die Tür am Kopfende seines Bettes. «Gregor », rief es - es war die Mutter -, « es ist drei Viertel sieben. » (28)

Comme il retournait en toute hâte ces pensées dans sa tête sans pouvoir se décider à quitter son lit, on frappa prudemment à la porte située à côté de son chevet [...]. «Gregor ! » disait-on - c'était sa mère - « il est sept heures moins le quart. » (29) française, mais on peut dire que l'allemand bégaie discrètement. Le " es » agit comme le bourdon d'une impersonnalité plus entêtée que ce qui se manifeste dans les 
modulations du français. Pour commencer, « on » ne se substitue pas parfaitement aux deux premières occurrences, car comme l'atteste l'étymologie, il suppose un agent humain - tout comme le pronom " man ", à l'étymologie similaire ${ }^{12}$ et contrairement à « es » qui est indéterminé également quant à la nature de l'agent, pouvant renvoyer aussi à un agent inhumain, animal, abstrait ou à un processus. Quand son identité est éclaircie ("Gregor ", rief es - es war die Mutter »), la proposition peut aussi bien être retournée, la formulation symétrique («die Mutter war es») étant également acceptable dans la langue : la mère était " ça » qui toquait et appelait. Le dernier " es » renforce encore la suggestion d'un processus, pour parler comme Deleuze, qui déborde entièrement le personnage, de manière irrépressible - peut-on faire qu'il (« es ») ne soit pas l'heure qu'il est, heure déjà tardive dans la transformation en cours?

L'analyse gagnerait à être poussée plus avant, mais pour notre propos disons qu'il serait possible de montrer comment ce « es", d'une correction exemplaire, hante de bout en bout la langue de La Métamorphose, qui se joue précisément sur le seuil où se trouve Gregor entre l'humain et ce qui ne peut plus se qualifier de tel. À un moment décisif, quand la sœur est sur le point de renier son frère, on note une hésitation encore entre le «er", humain, trop humain, et le "es" ("ça », cette créature monstrueuse pas même animale - «dieses Untier »). Or c'est après la mort de Gregor, quand par un processus irrépressible, il est effectivement devenu « es » - « es ist krepiert », « la bête est crevée », claironne la femme de ménage -, que Grete va pouvoir à son tour se métamorphoser en jeune femme ${ }^{13}$. De GREgor à GREte en passant par « es ", la langue aura effectivement bégayé. Sur ce point, elle est bien comparable au piaulement de Gregor, à propos duquel il est dit qu'au premier moment on y reconnaissait les mots sans équivoque et qu'ensuite tout se brouillait au point de faire douter qu'on ait bien entendu (29) - ou lu.

41 Le piaulement, c'est encore un nom pour le bégaiement, le silence ou l'enfance ${ }^{14}$, "l'affect de la langue» (Deleuze, 1993,139), qui hante la langue sans pour autant se laisser saisir en paroles agrammaticales, qui pousse le langage « à sa limite propre pour en découvrir le Dehors » (Deleuze, 1993, 94). Ici se loge le malentendu pour Rancière qui, là où Deleuze entend "résonner des anomalies", reste sensible à «la sourde présence de la forme insolite qui continue à hanter la langue de Bartleby » (Deleuze, 1993, 90 ; je souligne), ne perçoit que des « constructions normales », selon une norme qu'il associe à Flaubert, et ne se sent pas tenté de tendre l'oreille. Deleuze, par ailleurs, ne se distingue pas non plus par une lecture très serrée de "Bartleby ", pour revenir à notre préoccupation de départ, mais par son insistance sur les quasi-anomalies de la langue, il ouvre la voie à une critique plus soucieuse des effets textuels et à une mise à l'épreuve des hypothèses philosophiques par le texte littéraire, éventuellement portée par les objections soulevées par Rancière. Tel serait l'état des choses que la reprise rancièrienne de la formule permet de décrire.

Ce constat posé, savoir si le malentendu est un différend irréductible entre deux esthétiques et deux "politiques de la littérature » relève d'un autre questionnement. Nous l'aborderons plutôt à l'inverse en nous demandant si la formule de Bartleby, et plus généralement "Bartleby", est exemplaire de ce malentendu. Cela passe par un examen de la manière dont Rancière attrape la formule que lui fait miroiter Deleuze. La rapidité avec laquelle une équivalence est introduite entre elle et la maxime flaubertienne suggère qu'il ne l'attrape pas de la même manière que Deleuze. On dira plutôt qu'il tente de «se saisir » d'elle en la prenant dans les rets d'une autre, comme 
pour ne pas l'attraper telle une maladie d'amour dont son prédécesseur n'aurait su se prémunir et pour lancer à son égard un "I'd prefer him " que n'aurait pas renié Nippers. C'est pourtant déjà trop tard, car de toutes les formules de Deleuze dont il aurait pu s'emparer, c'est celle-là qu'il choisit. Quelle spécificité, alors, posséderait-elle et qu'est-ce que cela aurait à voir avec l'interprétation philosophique?

\section{Formule littéraire ou philosophique?}

Il semble qu'aucune discussion de "Bartleby » après Deleuze n'interroge l'emploi du terme «formule " pour qualifier le propos du personnage éponyme. Jacques Rancière, pour sa part, renchérit plutôt en faisant du titre-formule de Deleuze le point d'appui du sien et en subsumant la formule - celle de Deleuze ou celle de Bartleby? - sous une autre. Sous cet angle, le débat est entre formules et, qu'il le veuille ou non, Rancière a déjà validé la méthode de lecture deleuzienne. Il paraît curieux dès lors de parler de "formule littéraire». L'expression mérite d'être glosée. S'agit-il de la formule de Bartleby / «Bartleby », comme l'adjectif porte spontanément à le croire ? Il serait alors traité de l'interprétation philosophique d'une formule puisée dans la littérature, à condition de savoir ce que cela signifie et ce qui la distingue d'une expression ou d'un énoncé de même origine. Ou peut-être faut-il comprendre qu'il est fait référence, de manière plus oblique, à Deleuze comme philosophe littéraire, philosophe dont les formules ou concepts sont littéraires. En ce cas, que signifie cette insistance, cette hantise du littéraire dans le philosophique ? Est-ce l'image de la philosophie hantant ce qui la hante, attrapant ce qui l'attrape ? Enfin, il est loisible de comprendre l'adjectif relativement à l'objet dont s'occupe la formule deleuzienne: la formule qui expliquerait le phénomène littéraire aux yeux du philosophe. Cette dernière hypothèse est la seule qui soit traitée explicitement dans «Deleuze, Bartleby et la formule littéraire ", qui souligne une confusion entre ce que la formule dit et ce qu'elle fait, mais elle ne peut exclure les deux autres. L'indécision des termes reflète l'indécision du statut de la formule «I would prefer not to » ou, plus exactement, de l'énoncé «I would prefer not to » comme formule. En fait, la formule deleuzienne est littéraire dans les deux sens du terme : elle est issue de la littérature et toujours mêlée à elle en même temps qu'elle porte sur elle.

Revenons un peu sur nos pas. Au premier abord, la formule dont Deleuze parle est semblable à une formule magique, un philtre qui trouble tout et tout le monde. Elle s'oppose donc à une " formule ordinaire » (Deleuze, 1993, 89), c'est-à-dire une formule langagière qui ne dérange rien ni personne parce qu'elle est " consacrée ». Sa magie, au fond, est qu'elle est « une forme insolite ", « un trait d'expression qui contamine tout, s'échappant de la forme linguistique " (Deleuze, 1993, 100). Il y a un double paradoxe, ici. «I would prefer not to » est l'une de ces formes qui s'échappent de la forme ou, dit autrement, une formule de l'informulable. De ce point de vue, un énoncé devient formule parce qu'il fait bouger les cadres (autre sens de formula), à la limite. Sa capacité d'intriguer la philosophie pointe alors également un autre paradoxe : devient formule philosophique (formule pour la philosophie) ce qui est d'abord formule expressive, "d'une haute poéticité », et ce en raison, semble-t-il, de cette poéticité.

Voici ré-articulée l'antique opposition de la poésie et de la philosophie, que Maurice Blanchot représentait ainsi, dans L'Écriture du désastre, ce livre-même d'où Deleuze a pu tirer l'idée de préférence négative, dans les pages consacrées à «Bartleby»: «Le 
philosophe qui écrirait en poète viserait sa propre destruction. Et même la visant, il ne peut l'atteindre. La poésie est question pour la philosophie qui prétend lui donner une réponse, et ainsi la comprendre (la savoir). La philosophie qui met tout en question, achoppe à la poésie qui est la question qui lui échappe. » (Blanchot, 1980, 104) On dirait que, par amour de la formule et sans doute en souvenir de Nietzsche, Deleuze ne pouvait pas tout à fait renoncer à ne pas écrire en poète pour que la poésie subsiste comme question au sein de la philosophie, sans pour autant qu'il vise sa propre destruction. Dès lors, évoquer une «formule littéraire " pour référer à l'argument deleuzien est une bonne traduction de cette ambivalence.

Malgré tout, on constate que Rancière choisit rapidement de ne voir les choses que d'un seul côté, celui de la formule philosophique, suivant un argument faussement simple. Deleuze, est-il remarqué, privilégie le conte ("Bartleby », "La Métamorphose ») qui «est la formule magique qui raconte l'histoire d'une formule magique, qui métamorphose toute histoire de métamorphose en démonstration de sa puissance métamorphique ", d'où, chez Deleuze, « un jeu assez singulier entre ce que l'on appelait classiquement la forme et le contenu de l'œuvre " (Rancière, 1998, 188). Mais la singularité est aussi du côté du raisonnement de Rancière. Il mentionne l'opposition forme / contenu pour faire valoir que Deleuze au fond ne retient que le second («ce que la fable nous raconte») et, implicitement, que l'opérativité du conte vient de la langue ou de la forme ${ }^{15}$. Si l'on concède, par hypothèse, que la "magie » du texte est une question de forme, nous nous retrouvons face à une contradiction apparente. La formule racontée, contenue dans le récit, n'est en ce cas magique que nominalement et serait dès lors séparée de la magie qui la contient, autant par conséquent que du conte comme structure formelle. La formule magique est définie une fois comme efficace et forme et une autre fois comme objet de discours dépourvu d'effet et de forme, pur dit. Contée, elle ne serait pas touchée par la magie de la langue, ni en elle-même, ni comme prise dans l'ensemble de la fiction.

En d'autres termes, Rancière paraît exclure ce qu'il vient d'affirmer d'une autre façon : que la formule est magique et qu'en même temps elle ne l'est pas. Plus exactement, il fait glisser l'efficace d'une formule à l'autre, pour isoler et traduire philosophiquement la première dans les termes d'une autre. Il tombe ainsi dans le piège de la formule en ne remarquant pas que c'est la lecture d'abord qui fonde l'énoncé de Bartleby en formule et - c'est tout un - la formule littéraire ainsi constituée en formule philosophique, ou pour la philosophie. Il est temps d'interroger le choix du vocable introduit par Deleuze et largement repris depuis ${ }^{16}$, alors même que l'œuvre se contente de faire référence, de manière plus générale, à un "mot» - «I had got into the way of using the word "prefer" upon all sorts of not exactly suitable occasions » (81), «"So you have got the word, too," said I, slightly excited. » (81).

D'un côté, on peut y voir une reconnaissance de sa puissance, dans la mesure où le terme isole l'énoncé et évoque la magie, une combinaison d'éléments fondamentaux ou encore une mise en équation d'un devenir. Mais le revers de cet anoblissement est que sa littérarité s'y perd, pour des raisons que nous pouvons tenter d'approcher à partir des réflexions de Maurice Blanchot sur la parole de l'art et son interprétation. Pour lui, «le langage, dans le monde, est par excellence pouvoir». Il «nous jette dans la dialectique du maître et de l'esclave », en ce que par lui on peut nommer et nommer « est cette violence qui écarte ce qui est nommé pour l'avoir sous la forme commode d'un nom » (Blanchot, 1990, 48) - ou, dira-t-on, d'une formule. 
49 Mais une distinction est introduite entre le langage du poète et celui du maitre. On peut être tenté de les confondre, comme Nietzsche lorsqu'il affirme le « sérieux terrible » de l'art et son pouvoir. Une telle interprétation méconnaît ce qui est pour Blanchot l'exigence de la littérature en ce qu'elle n'est vue que «prise dans la dialectique du maître et de l'esclave » et "déjà devenue instrument de puissance » (Blanchot, 1990, 48). C'est au contraire de ressaisir «le lieu où le langage est encore relation sans pouvoir, langage du rapport nu, étranger à toute maîtrise et à toute servitude » qu'il s'agit. Il est facile de voir comment Deleuze lecteur de «Bartleby » prête le flanc à une telle critique, désireux qu'il est de voir dans l'énoncé qu'il isole un étendard de la révolte des frères, faisant dès lors basculer sa poéticité - le langage sans relation de pouvoir - dans la performativité du mot d'ordre. En somme, Deleuze trahirait son intérêt premier pour la langue dans le monde. "I would prefer not to » serait à la fois une expression de ce lieu de la langue sans relation de pouvoir, une langue qui comme son énonciateur préférerait ne pas être aux prises avec le monde et une des prises que la langue peut avoir sur le monde qui trouve là son efficace. Nulle surprise dès lors si nombre de philosophes depuis Deleuze ne sont pas allés plus loin que l'énoncé comme formule ayant à répondre du monde et ils y répondent depuis ce monde en en faisant une parole maîtresse.

Deleuze lui-même reste pris dans cette tension non dialectisable entre parole de poète et parole de maître, qui commence dès qu'est attribué le caractère de formule - que le terme soit utilisé ou non - à l'énoncé littéraire, et il semble toujours tenté d'en sortir par la porte du devenir tout en finissant par traduire la première parole en la deuxième. Ce qui fait que malgré tout la tension demeure est bien l'insistance sur les lignes de fuite comme dans un effort inverse pour compenser la transformation inévitable et "faire de la parole maîtresse une parole sans maître " (Blanchot, 1990, 49). Reste qu'il n'y a pas de confrontation prolongée au texte chez Deleuze, comme si ce qui devient pour lui la formule était un pare-feu contre la poéticité de la langue qu'il fait miroiter sans plus de précisions. Cette absence est non moins remarquable chez Rancière qui, s'il la fait remarquer chez son prédécesseur, ne propose pas pour autant une lecture plus rapprochée de l'œuvre dont il se tient à distance redoublée par le renvoi à une deuxième formule. Cet évitement paradoxal renchérit de facto sur la critique adressée à Deleuze de n'être au fond pas assez soucieux du poétique mais on évitera d'en conclure que les deux philosophes manquent l'objet littéraire parce qu'ils négligent purement et simplement les phénomènes dans leur littérarité, ne les comprenant que comme philosophèmes. Il convient au contraire d'affirmer que leur lecture pèche d'être trop peu philosophique, c'est-à-dire métacritique, en ne mettant pas leurs arguments à l'épreuve des textes - ce qui n'est envisageable que par un engagement de lecture plus soutenu, au risque d'une crise de la parole maîtresse philosophique.

51 À rebours de l'hypothèse commune, nous n'opposerons donc pas la philosophie et la critique littéraire, laquelle serait entendue comme critique textuelle, au nom d'une fidélité supposée à l'œuvre. La mé-prise philosophique, si c'est cela dont il est question, peut éclairer la question de l'interprétation littéraire, car elle n'est pas le privilège ou la malédiction de la seule philosophie. Il n'est pas nécessaire en effet de «nommer » l'énoncé littéraire, la " parole de poète », par quelque nom pour qu'il puisse servir une parole maîtresse. Toute lecture, en ce qu'elle procède inévitablement par soustraction, analyse, est soumise à l'aporie que l'on cherche à réduire par une stricte opposition de 
la philosophie et de la poésie (ou de son représentant le plus fidèle: la critique littéraire). Le piège de "Bartleby" où se laisse prendre chaque lecture est que la nouvelle isole de façon manifeste l'énoncé qui semble devoir la résumer et que l'interprétation paraît ne faire que répéter (amoureusement ou non) ce qui est déjà aphorisme ou phrase allusive.

«La phrase isolée, aphoristique, » écrit Blanchot :

attire parce qu'elle affirme définitivement, comme si plus rien ne parlait autour d'elle, en dehors d'elle. La phrase allusive, isolée aussi, disant, ne disant pas, effaçant ce qu'elle dit en même temps qu'elle le dit, fait de l'ambiguïté une valeur. «Mettons que je n'ai rien dit.» La première est normative. La seconde croit échapper à l'illusion du vrai, mais se prend à l'illusion même comme vrai, croit que ce qui a été écrit peut se retenir. (Blanchot, 1980, 203)

Rancière verrait donc dans "I would prefer not to " plutôt un aphorisme qu'il préfère reprendre en termes flaubertiens mais sans justifier la valeur qui lui est accordée par un approfondissement de l'œuvre. Deleuze serait plus réceptif à sa valeur allusive, sa façon de dire et en même temps de ne pas dire, mais sans pouvoir échapper à l'illusion du vrai ou du moins du pouvoir de la parole poétique dans le monde, oscillant vraisemblablement entre deux positions. Mais c'est le sort de toute lecture qui prendrait prioritairement appui sur ce par quoi l'œuvre si ouvertement l'appâte que de risquer de manquer le lieu sans pouvoir qui fait l'enjeu de la littérature. Et que serait une lecture de "Bartleby" qui préférerait ne pas s'attarder sur «la formule de sa gloire»? Par la réitération de sa préférence négative elle pourrait bien être poétiquement la plus proche de l'œuvre, quitte cependant à y disparaître comme discours critique et à perdre la possibilité de la mettre en question(s). Le choix n'est pas dès lors de faire ou de ne pas faire de l'énoncé littéraire une formule, ou telle ou telle formule, il est de le saisir dans ses devenirs, y compris philosophiques au sens plus restreint, et de ne pas « l'attraper » à l'exclusion de toute autre formule potentielle.

En effet, d'autres événements textuels, qui ne sont pas non plus sans lien avec «le lieu où le langage est encore relation sans pouvoir » ni pour autant sans efficace, requièrent une attention critique. Nous n'en retiendrons que quelques-uns avant de conclure. Un premier exemple concerne en l'occurrence une variation de l'énoncé de Bartleby que nous avons déjà remarquée. Nommer «la » formule revient en grande partie à tenir à l'écart d'autres formules proches. La chose est flagrante chez Rancière qui les subsume toutes dans sa traduction, mais Deleuze pour sa part, on l'a vu également, ne va pas plus loin qu'une liste rapidement commentée. Or, considérons le «I'd prefer him, if I were you " de Nippers. Même s'il ne se conçoit pas indépendamment des mots prononcés par Bartleby, il a une force singulière, quasi agrammaticale elle aussi. La phrase est grammaticale, bien qu'incomplète quant au sens, s'il s'agit de préférer quelqu'un (Bartleby, donc) à quelqu'un d'autre (non mentionné). Elle ne le serait plus s'il s'agissait d'élider ce que Nippers aimerait que Bartleby fasse - la formulation correcte serait alors «I'd prefer him to », suivi ou non d'un syntagme verbal. Mais dans l'usage qu'en fait Nippers, la langue bouge en même temps qu'elle bégaie en passant d'un locuteur à l'autre. Les mots repris s'autonomisent, coupés de leur fonction d'expression habituelle (aimer plus quelqu'un ou quelque chose que quelqu'un ou quelque chose d'autre) pour faire de « prefer » un verbe d'action.

Sans chercher, comme son employeur, à explorer la sémantique ou le symbolisme de l'énoncé initial, Nippers, en fait, en a saisi la performativité qu'il retourne à l'envoyeur sous forme de menace, prefer devenant, en termes généraux, synonyme de «s'en 
prendre à » ou " en remontrer à » ou encore, si l'on veut, laissant entendre l'écho familier d'un verbe chargé de sens dans le milieu légal, " déférer devant les tribunaux » (refer). L'employé belliqueux - c'est son humeur du matin - dit en creux le bouleversement suscité par ces mots tant répétés par Bartleby en même temps que son énoncé ravage la langue en utilisant un verbe de sentiment positif pour évoquer une action agressive. Ainsi voit-on sur ce point confirmé l'argument de Deleuze concernant les dérangements causés à la langue et contestée l'objection de Rancière, car le trouble n'est pas seulement perceptible au niveau diégétique ou de la fable, l'inventivité linguistique ne se laissant pas restreindre à la seule chaîne narrative.

À l'inverse, les hypothèses de Deleuze sont en partie prises en défaut lorsqu'il se débarrasse d'un revers de la main d'un autre énoncé qui sonne comme une formule, à savoir les mots de conclusion prononcés par l'homme de loi : "Ah, Bartleby! Ah, humanity!» (99). À ses yeux, ils constituent «non pas une connexion, mais au contraire une alternative où il [l'homme de loi] a dû choisir contre Bartleby la loi trop humaine » (Deleuze, 1993, 104). Le problème avec cette interprétation n'est pas que Deleuze l'ait choisie contre une autre peut-être plus favorable à la figure paternelle ou, à tout le moins, à l'homme de loi, ou contre une autre plus neutre ; le problème est qu'il ait pu interpréter avec autant d'assurance ces mots qui sont au moins aussi énigmatiques. Il ne peut le faire, et de cette manière-là, qu'à partir de sa formule princeps de l'émancipation schizophrénique des fils et en passant par-dessus tous les silences thématiques et structurels du récit. Il est en soi très difficile de comprendre le sens ou plus généralement la fonction de deux exclamatives réduites à une onomatopée et un nom, et tout aussi malaisé de décider de leur lien, si même il y a un lien. En outre, ni le contexte immédiat, ni le texte dans son ensemble ne nous permettent de trancher dans ces questions. Il existe en l'occurrence un contraste saisissant entre ces exclamations brutes, affectuelles et les phrases jusque-là rigoureusement construites et appliquées qui soutiennent le compte rendu du narrateur.

La formule choisie par Deleuze, et Rancière après lui, en masque donc une autre tout aussi incomplète et quasi agrammaticale par sa syntaxe tronquée, et partant aussi ravageuse pour le langage autrement ordonné du récit de l'homme de loi. Les derniers mots de la nouvelle seraient de fait également susceptibles de tenir lieu de formule de la littérature, mais à condition de changer de perspective de lecture pour intégrer celle du narrateur-homme de loi, c'est-à-dire de tenter d'approcher une formule du récit qui touche à sa condition de possibilité. Il est à remarquer d'abord que la figure du protagoniste lecteur et interprète est souvent laissée dans l'ombre des commentaires de "Bartleby ", quand bien même elle appartient de plein droit à la scène de l'écriture. L'homme de loi en effet hésite lui aussi entre traduire l'énoncé de son scribe en formule maitresse ou la comprendre dans les termes d'une autre («the doctrine of assumptions " par exemple, ou la providence divine) et, d'un autre côté, se laisser prendre par sa magie - «Somehow, of late, I had got into the way of involuntarily using the word 'prefer' upon all sorts of not exactly suitable occasions. " (81). Cette tension, qui concerne à la fois la lecture des événements de la diégèse et leur mise en mots, est le moteur de son écriture, bien qu'il soit - autre tension - plus porté par métier aux écritures juridiques, dont le pouvoir est bien ordonné. Il faudrait également s'intéresser de près à cette formule tout autant incalculable et indémêlable de celle juste évoquée qui nous fait signe à partir de la rumeur autour du service des dead letters dans l'étrange épilogue de la nouvelle. Il nous faudrait prendre le temps de démontrer comment l'homme de loi, en tant qu'employeur et que narrateur, devient le 
destinataire de ces lettres qui auraient (le conditionnel est de mise) bouleversé Bartleby, le destinataire de la lettre morte qu'est Bartleby - ou peut-être vaudrait-il mieux dire, au vu de la surenchère critique, cette lettre à l'encan dont «Bartleby » est le nom.

De ce point de vue, l'énoncé de Nippers devient un révélateur ironique et non plus un avatar secondaire d'une formule princeps. N'oublions pas qu'il s'adresse explicitement à son employeur : «I'd prefer him, if I were you ! ». Il exprime l'embarras où l'homme de loi se trouve. Il n'arrive pas à préférer Bartleby au sens où Nippers l'entend, le préférer comme il le ferait s'il était à sa place. Il lui arrive d'y penser mais, malgré tout, tout se passe comme si il préférait ne pas "préférer » Bartleby. Et c'est pourtant ce qu'il fait, comme s'il suivait un conseil que Nippers ne sait pas qu'il vient de lui donner. Il préférera Bartleby à Nippers effectivement, demandant poliment à ce dernier de se retirer - «'Mr Nippers,' I said, 'I'd prefer that you would withdraw for the present.' «(81) - , ce qui lui fera craindre que « le mot », qui l'a déjà contaminé, ne l'ait affecté mentalement et finisse par produire d'autres aberrations, dont il ne dit pas si sa transformation d'homme de loi en homme de lettres fait partie.

Cette esquisse montre les limites d'une interprétation à coup de formule, quelle que soit celle que l'on choisit ou, semble-t-il, qui nous choisit et quelles que soient donc celles que l'on occulte ainsi. On aura compris que cela est inévitable mais non réservé à la philosophie. Comme on l'a vu avec nos deux exemples, la lecture formulaïque de la philosophie cependant met l'accent sur les limites d'une telle lecture, dès lors qu'elle oublie que toute formule interprétative est à la fois littéraire et philosophique, ce qui est encore plus patent lorsqu'elle est plus éloignée de son contexte d'origine par une lecture de second degré (Rancière commentant Deleuze).

La littérature pose toujours question pour la philosophie, l'aidant même à formuler ses questions. Par là, elle justifie l'intérêt de la philosophie pour ses productions. «Bartleby » en ce sens est exemplaire pour la façon dont ce récit lui tend la perche en mettant en scène l'interprétation d'une énigme littéraire - n'oublions pas que le narrateur se lamente que l'absence de tout matériau biographique sur Bartleby est une perte irréparable pour la littérature (59). L'interprète est un philosophe, trop confiant d'abord en ses formules, les formules philosophiques et religieuses de son temps, qui se hasarde dans les parages du poétique. Il attrape donc, aux deux sens du terme, la formule littéraire, au-delà même de l'énoncé de Bartleby et, suspendu entre philosophie et littérature, reste indécis, comme en témoignent ses hésitations sur le qualificatif le plus approprié à son texte - «history», "meagre recital» ou «little narrative » (99)? - ou sur l'opportunité de propager « one little item of rumor », ce «vague report» (99) concernant le présumé emploi au Dead Letter Office. Sa justification sur ce dernier point est d'ailleurs significativement embarrassée, comme s'il ne savait toujours pas ce qui le pousse à vouloir parler de Bartleby : « But, inasmuch as this vague report has not been without a certain suggestive interest to me, however said, it may prove the same with some others ; and so I will briefly mention it. » (99) On aura noté la double négation (not without) et l'ambivalence de l'adjectif (certain) qui dit à la fois le flou quant à la nature de l'intérêt porté à la rumeur et la certitude de son pouvoir de suggestion. Tout cela pour motiver la mention de ce bruit dont le narrateur ne peut établir (ascertain) le bien-fondé : pour motiver, donc, son entrée dans la fiction.

Dès lors que la perspective de lecture inclut celle de l'énonciation, il apparaît que la force de la nouvelle va au-delà de ce que crée la formule préférée des philosophes et 
suggère une formule d'une complexité bien plus grande puisque s'y retrouvent la poéticité de l'énoncé de Bartleby et bien d'autres mais aussi un dispositif énonciatif et herméneutique contaminé par cette poéticité. On peut donc comprendre la séduction de la formule de Bartleby chez les philosophes également comme une sorte de mécanisme de défense contre la complexité de la formule fictionnelle, plus dérangeante pour le philosophe, comme le donne à penser le portrait souvent peu flatteur donné de l'homme de loi, quand il n'est pas simplement ignoré, comme un gêneur. Il se pourrait bien en effet que le narrateur qui ne sait pas nommer une certaine fascination, cette fascination certaine qui le saisit, soit le portrait du philosophe ou du critique en homme de loi délogé de ses premises (son terrain d'action comme ses présupposés) par la question qui lui échappe. Et condamné comme lui à attraper la formule.

\section{BIBLIOGRAPHIE}

BERKMAN, Gisèle, L'Effet Bartleby, Fictions pensantes, Paris, Hermann, 2011.

BLANCHOT, Maurice, L'Écriture du désastre, Paris, Gallimard, 1980.

---, Le Livre à venir (1959), Folio, Paris, Gallimard, 1990.

DELEUZE, Gilles, Critique et clinique, Paradoxe, Paris, Minuit, 1993.

---, Francis Bacon : logiques de la sensation, La Vue le Texte, Paris, La Différence, 1996.

DELEUZE, Gilles et Félix GUATTARI, Qu'est-ce que la philosophie ? Critique, Paris, Minuit, 1991.

KAFKA, Franz, La Métamorphose / Die Verwandlung (1915), Folio Bilingue, Paris, Gallimard, 1991.

MELVILLE, Herman, « Bartleby », (1856), Billy Budd, Sailor, and Other Stories, Harmondsworth, Penguin Books, 1970, 57-99.

PEDOT, Richard, Le Seuil de la fiction : essai sur le secret, Paris, Michel Houdiard, 2010.

---, « Signer Bartleby : le devenir-personnage de la philosophie », L'Atelier [Journal en ligne], 2.1, 2010, http://revues.u-paris10.fr/index.php/latelier/article/view/49.

RANCIÈRE, Jacques, La Chair des mots : politiques de l'écriture, Incises, Paris, Galilée, 1998.

\section{NOTES}

1. Cf. aussi «Bartleby, ou la formule », 104-105 où le roman anglais et français pâtissent de la comparaison avec le roman américain et russe.

2. Qui savait lui aussi faire filer le langage par la formule. rappelons que Bleak House, avec son fameux «Circumlocution Office», a fini de paraître vers la fin 1853, peu de temps avant que la première des deux livraisons de «Bartleby » soit publiée.

3. Cf. Pedot, Le Seuil de la fiction, 33-40.

4. Cf. « La Littérature et la vie » (Deleuze, 1993, 11-17).

5. Cf. Deleuze et Guattari, Qu'est-ce que la philosophie? 
6. N'oublions pas la position critique de Critique et clinique vis-à-vis de la psychanalyse (œdipienne).

7. Deleuze allant même jusqu'à suggérer une passion homosexuelle.

8. Notons au passage que Rancière, de manière que l'on peut qualifier d'insidieuse, expose plus les torts du «deleuzisme » que ceux de la philosophie de Deleuze, dont il souligne souvent la cohérence.

9. Ce propos, même s'agissant d'une formule aussi célèbre, mériterait discussion.

10. Question posée à propos de Francis Bacon (Deleuze, 1996, 40)

11. Oubliant par ailleurs l'exemple de Cummings, qui servirait moins bien sa démonstration.

12. On étymologiquement vient d'homo ; man de Mann (homme).

13. Pour un commentaire plus détaillé de la question, cf. Pedot, 2010, 60-65.

14. Deleuze ne renvoie pas par hasard à Lyotard à propos de ce concept dans la dernière note de « Bégaya-t-il » (Deleuze, 1993).

15. En d'autres termes, il renforce l'opposition classique sans imaginer que forme et contenu puissent participer tous deux, y compris, et peut-être surtout, aporétiquement, à l'opérativité du texte.

16. $Y$ compris par l'auteur de ces lignes.

\section{RÉSUMÉS}

Cet article interroge la manière dont la philosophie lit la fiction, à travers l'exemple de «Bartleby », interprété par Deleuze dans son célèbre « Bartleby, ou la formule », commentaire à son tour livré à la critique d'un autre philosophe, Jacques Rancière. Il s'agit en l'occurrence de réfléchir à la façon dont la philosophie se laisse piéger par la formule de Bartleby / «Bartleby », incapable qu'elle est de décider entre formule poétique et formule philosophique.

This paper focuses on philosophical reading of fiction, namely "Bartleby the Scrivener", famously interpreted by Deleuze in his "Bartleby, ou la formule", which is in turn criticized by Jacques Rancière. It purports to show how philosophy is seduced by Bartleby / "Bartleby"'s formula, being unable to tell apart poetical and philosophical formulæ.

INDEX

Mots-clés : Melville, Hermann ; « Bartleby »; Interprétation philosophique ; Deleuze, Gilles ; Rancière, Jacques

Keywords : Melville, Hermann ; « Bartleby »; Philosophical interpretation ; Deleuze, Gilles ; Rancière, Jacques

\section{AUTEUR}

\section{RICHARD PEDOT}

Université de Paris Ouest 